\title{
Identifying and mitigating Sudden Unexpected Death in Epilepsy (SUDEP) risk factors
}

\begin{abstract}
Introduction

Sudden Unexpected Death in Epilepsy (SUDEP) is a significant cause of death for people with chronic epilepsy. Good practice guidance in the UK and the USA expect SUDEP to be discussed with the individual. The event rarity, methodological variance and lack of robust research into the pathological mechanisms, associated risk factors, and management strategies have created a challenge on how and what to discuss. There are some significant associations which allows for risk assessment and mitigation.

Areas covered

We review the current understanding of static and modifiable risk factors for SUDEP and how to manage these more effectively. Longitudinal risk may be assessed using standardised risk assessment tools which help in communicating risk. Technological advancement allows measurement of physiological parameters associated with seizures and risk of SUDEP using small wearable devices. Further evidence is needed to demonstrate such technologies are efficacious and safe.

Expert commentary

Risk reduction should be an important part of epilepsy management and we suggest a Gold Standard of Care which healthcare professionals and services should aim for when approaching SUDEP risk management. A Minimum Standard of Care is also proposed that is practical to implement, that all people with epilepsy should expect to receive.
\end{abstract}

\section{Key words}

Mechanism; Risk Factors; avoidable death, preventable death 


\section{Introduction}

The prevalence of active epilepsy ranges between 0.6 and 1\% [1]. A diagnosis of epilepsy comes with increased rates of co-morbidity and mortality from all causes [2]. In a UK epilepsy population only $52 \%$ were seizure free and this has a direct relationship with risk [3]. For people with symptomatic epilepsy life expectancy may be up to 10 years lower than the general population [4]. This increased mortality is related to the underlying cause of epilepsy, and seizures themselves including status epilepticus [5]. It is widely regarded that the most common cause of death in the population with chronic epilepsy is sudden unexpected death in epilepsy (SUDEP) [6].

\section{Incidence}

The reported incidence rates of SUDEP range widely from 0.09 to 9.3 per 1000 person-years [7]. The identification of SUDEP cases in community samples and epilepsy cohorts has largely been retrospective in nature, involving review of data from hospital and autopsy records. A large population based study of people with epilepsy in the USA reported an estimated incidence of 0.35 cases per 1,000 person years. This gives a Standardized Mortality ratio of nearly 24 times that of the general population [8]. Similar results have been replicated in a nationwide Danish population study [9]. Other studies have reported significantly higher rates of SUDEP in the epilepsy population. The reason for such variance in incidence rates is likely related to case selection and methodological differences in study design. A more recent systematic review of the literature estimates a crude annual incidence rate of $1.16(0.95-1.36)$ per 1000 person years [10].

In pooled data it can be seen that the incidence of SUDEP is higher in cohorts of people with treatment resistant epilepsy, uncontrolled convulsions, those treated at specialist epilepsy centres, the epilepsy surgery population [11] and those with nocturnal seizures [12]. A nationwide population-based cohort study in Sweden of nearly 60,000 people with epilepsy identified 99 deaths meeting SUDEP criteria [13]. It accounted for about $5 \%$ of deaths but this was increased to $36 \%$ in the population 15 years or below. This is in contrast to previous suggestions that SUDEP risk may be lower in children [14, 15, and 16]. This study highlights the difficulty in case ascertainment as only around two-thirds of SUDEP cases had epilepsy recorded on the death certificate, suggesting that current incidence may be underestimated [13].

\section{Definition}

SUDEP has been defined as "the sudden, unexpected, witnessed or unwitnessed, non-traumatic, and non-drowning death of people with epilepsy with or without the evidence of a seizure, excluding status epilepticus, and in which post-mortem examination does not reveal a structural or toxicological cause of death" $[17,18]$. Making a diagnosis of SUDEP is not straight forward. To date there are no diagnostic tests available and even after post-mortem examination SUDEP is largely a diagnosis of exclusion. As a result establishing caseness can be challenging. In 2012 a unified SUDEP definition with seven classifications was proposed to facilitate case definition in clinical and research 
work [19] (Appendix 1) in view of the variability in access to routine post-mortem examinations and lack of standardised recording of findings.

\section{Pathophysiology}

To manage SUDEP risk effectively it is important to understand the pathophysiology involved. There has long been debate over whether the primary mechanism in SUDEP is derived from cardiac or respiratory dysfunction. It is established that seizures can effect cardiac functioning, leading to arrhythmias and abnormalities in blood pressure [20]. Post-ictal cardiac arrhythmias have been found to occur most commonly following convulsions and are associated with near SUDEP cases [21]. It is also known that respiratory depression and apnoea can occur in the peri-ictal and post-ictal period $[22,23]$. A recent review suggests that SUDEP cases are often characterized by post-ictal apnoea and bradycardia which progresses to asystole. The identification of post-ictal generalised EEG suppression may potentially offer a biomarker for brainstem dysfunction which is thought to form part of the pathway to SUDEP [24]. The current informed view is that SUDEP is a culmination of a multifactorial mechanism involving numerous systems causing a perfect physiological storm (appendix 2). Cardiac arrhythmia, respiratory dysfunction, dysregulation of systemic or cerebral circulation \& seizure-induced hormonal and metabolic changes are suggested as potential pathological mechanisms most probably triggered by the peri-ictal concurrence of a number of predisposing and precipitating factors [25]. Similarities can be drawn to Sudden Cardiac Death where structural cardiac abnormality and genetic variation can predispose to sudden death.

The MORTEMUS study [26] confirmed some important characteristics of SUDEP in 16 cases of definite/probable SUDEP, and 9 cases of near-SUDEP in monitored individuals (Appendix 3). Results indicate that there is likely to be a centrally mediated compromise in cardiac and respiratory function following a convulsion. Terminal apnoea occurred prior to asystole in all cases [26]. Most SUDEP cases are unwitnessed and supervision itself may act as a protective factor 27,28$]$. Where SUDEP has been witnessed it is usually preceded by seizure activity [29].

Postictal generalized electroencephalographic suppression (PGES) which is commonly associated with generalised seizures in adults is strongly associated with severe hypoxemia. There is recognition of primary pulmonary problems rather than hypoventilation influencing respiratory depression in seizures and being linked to the duration of the seizures [30] and correlating to contralateral hemisphere spread. Studies have shown central apnoea in three fifth of people with seizures and two fifth of all seizures in addition to oxygen desaturation levels fall below $85 \%$ in one fifth of all seizures [31]. Moreover, oxygen desaturations below $85 \%$ were associated with the rise in the endtidal $\mathrm{CO}_{2}$ that in some patients persisted despite an increase in respiratory rate [20], thus indicating a primary pulmonary dysfunction rather than hypoventilation.

In up to two fifth of people with refractory epilepsy cardio autonomic concerns have been noticed [32]. Impairments in vagal driven activity of Heart rate variability were consistently observed in people with epilepsy with an association of more profound changes with refractory epilepsy [33].

Further the genetic mechanisms underlying cardiac and pulmonary factors have been explored. Altered expression of serotonin linked genes which have a role in seizures, respiration and arousal have been of interest in animal models but the findings have not yet been successfully replicated to 
humans [34]. Similar animal model studies on some novel candidate genes particularly those influencing ion channels are implicated in developing cardiac arrhythmias exacerbations in seizures due to vagal hyper excitability.

A polymorphic model which involves multiple potential pathways with various entry points for a terminal event has been postulated as leading to the final lethal pathway of SUDEP [32].

\section{SUDEP Risk Factors}

Investigations into the risk factors for SUDEP are heterogeneous in terms of population and methodology so it can be difficult to interpret the relevance of findings in individual studies, and there are often contradictions. Attempting to identify 'certain' risk factors in such a complex multifactorial condition may depend upon constructing robust prospective investigations. This is not straight forward when we do not fully understand the pathological mechanisms involved and when events are so rare. The risk factors identified within individual studies should form part of our ever growing knowledge of how to identify and manage risk in the epilepsy population. Pooling the information available can reduce any conflict and help interpretation to produce a broad base of potential risk factors to consider when communicating with individuals (Table 1).

\subsection{Static risk factors}

There are a number of demographic factors that are linked to an increased risk of SUDEP. Male gender and early onset of seizures ( $<16$ years at onset) confer statistically significant risk. The duration of epilepsy for more than 15 years has also been identified as a significant risk factor [35, 36]. SUDEP can occur at any age but some circumstantial evidence suggests that the highest risk lies between the ages of 20 and 40 years [37].

It is well recognised that people with epilepsy and intellectual disability have higher rates of complex treatment resistant epilepsy and co-morbidities. A cohort of pupils at a residential school for people with intellectual disabilities showed a high mortality ratio with the majority of deaths considered epilepsy related and half of the death described as sudden. All sudden deaths occurred when pupils were not under close supervision [27]. Intellectual disability (ID) may be a significant independent risk factor for SUDEP with an adjusted odds ratio of 4.6 (1.2-1.8) [38]. In other population samples SUDEP has been identified as the second most common cause of death in people with ID and epilepsy, and rates of SUDEP found to be significantly higher in comparison to the general population [39]. When people with ID and epilepsy receive appropriate specialist care this correlation has not been seen. Therefore it may be the lack of access to specialist services that plays a key role in increasing risk for this population [40].

\subsection{Genetics}

There are certain genetic mutations that are associated with an increased SUDEP risk. Evidence emerging suggests that candidate genes are increasing and that there appears to be an overlap with some of the candidate genes for sudden cardiac death [41]. One example is the association with long QT syndrome (LQTS). One group has undertaken an exome-based analysis of SUDEP cases and 
identified known and candidate variants predisposing to cardiac arrhythmia [42, 43]. These findings may represent a sub-population of SUDEP cases.

Dravet syndrome is an epileptic encephalopathy characterised by the emergence of severe febrile seizures within the first year of life. Later individuals develop multiple treatment resistant seizure types, slowing in developmental progression and cognitive decline [44]. Most affected people have mutations in the SCN1A gene [45]. This gene encodes for part of the voltage-gated sodium channel and plays an important role in brain and cardiac functioning. People with Dravet Syndrome have a predisposition to depressed heart rate variability and mouse models also suggest prolonged ictalonset of bradycardia $[46,47]$. This may play a role in the significantly increased risk of premature death in Dravet syndrome $[48,49]$.

\subsection{Modifiable Risk Factors}

\subsubsection{Seizure profile}

A case control study in the 90's was one of the first to identify that those who a high frequency of seizures were more at risk of SUDEP. In a pooled analysis of cohort studies examining the epilepsy population it was shown that experiencing three or more convulsions within a year significantly increases risk of SUDEP [35]. When people have up to 20 convulsions in a 3 month period the adjusted odds ratio may be as high as 20 [28]. Having a high seizure frequency regardless of seizure type is also a notable SUDEP risk factor [50]. Consistently, regardless of study design or population, convulsions seem a key risk factor [51].

Nocturnal seizures may independently increase the SUDEP risk 3-fold [12]. There is an argument that this may in part be due to lack of appropriate supervision. It is known that nocturnal supervision seems to provide protection against SUDEP [28]. The MORTEMUS study clearly describes delayed resuscitation when events occurred outside regular working hours, a time of lower staff monitoring. This delayed response was associated with a significantly increased risk [26]. A recent Cochrane review [52] suggests that there is some evidence to support the employment of monitoring device. Epileptologists should discuss night-time observation and other overnight monitoring techniques, including remote listening devices, to reduce the risk of SUDEP in people with frequent convulsions particularly if nocturnal. Privacy issues, the burden on family members, and other psychosocial circumstances also need to be considered [53].

There is a suggestion of an association between sleeping in the 'prone position' and identified SUDEP cases $[26,40]$. A systematic review and meta-analysis confirms this correlation which is particularly evident in those under the age of 40 years [54]. A disparity has been observed in the sleeping position in cases of SUDEP compared to non-fatal convulsions in a sample supervised by video-EEG [55]. Modifying this risk is not straightforward as position may change during a convulsive seizureeven if the individual does not begin in a prone position they may be end up prone during the course of the seizure.

\subsubsection{Anti-epileptic drugs}


The higher the number of anti-epileptic drugs an individual is prescribed the higher the risk of SUDEP observed $[28,38,56]$. Findings suggest a relationship between the number of convulsions, number of AEDs, and risk of SUDEP [35]. The prescription of multiple AEDs may be a surrogate for poor seizure control. One study suggested polytherapy to be a significant independent risk factor for SUDEP after adjustment for confounders including seizure frequency. This may be related to potential cardiac effects of the medication [57] , however, these findings have not been replicated [35]. It has been shown that no AED treatment or unclear treatment history does put individuals with epilepsy at increased risk of SUDEP [58].

There is some evidence to suggest that changes in treatment or dose of AEDs may place people at increased risk [56, 59]. Review of post-mortem blood levels of AEDs also indicate that noncompliance with prescribed AEDs may be a precipitating factor, however there are real difficulties establishing non-compliance and these findings have not been confirmed [35]. Several studies have indicated that there is no suggestion of lower compliance rates in SUDEP cases compared to death in epilepsy of other causes [60]. There is no conclusive evidence that any specific AED is associated with an increased risk of SUDEP though there had been some speculation of the role of carbamazepine and subsequently lamotrigine [51]. There is some evidence that an adjunctive new AED treatment in those with treatment resistant epilepsy reduces SUDEP incidence by around six times compared to that observed on placebo [61].

\subsubsection{Co-morbidities}

\section{Psychological}

A population-based study examining the risk factors of all causes of premature mortality in epilepsy suggest that co-morbid mental health problems such as depression may increase mortality risk [62]. There is also evidence from mouse models to suggest that treatment with selective serotonin reuptake inhibitors may reduce the risk of sudden death suggesting that serotonin may play a role in the sudden death pathway [63].

Results from a recent Swedish nationwide population-based cohort study suggest that any psychiatric co-morbidity may increase SUDEP risk. In females this risk may be five folds than of individuals with no psychiatric co-morbidity. This study does not control for some of the complex issues associated with psychiatric illness including engagement with services [13]. It has also been observed that treatment with anxiolytic and antipsychotic medication specifically confers an increased risk of SUDEP, however these findings have not since been replicated [56]. In context these findings conflict with a previous systematic review that identified no association between an increased risk of SUDEP and a broad range of psychiatric presentations and treatments [64]. The comorbid use of alcohol has often been suggested to increase risk of mortality in epilepsy including SUDEP $[40,62]$.

\section{Physical health}

The concept of SUDEP plus has been advanced into the criteria for diagnostic consideration [18]. Previously, a diagnosis of SUDEP was made when there were no other significant co-morbidities 
contributing to the death. More recently diagnoses of SUDEP are being made in the context of multi-morbidity. An example of this is the consideration of Takotsubo syndrome (TKS), an acute cardiomyopathy that may arise following stressful insults including seizures [65].

\section{Reducing SUDEP risk}

Risk reduction measures should be targeted at the identified risk factors. There is evidence to support the need to ensure seizure control is maximised, particularly in regard to reducing convulsions. This should include medical and non-pharmacological interventions including epilepsy surgery, VNS, RNS and diet if appropriate. There are also other important aspects of risk management to consider including ensuring adequate supervision, particularly at night, to minimise the risk of airway obstruction and cardiorespiratory distress.

The Cochrane review investigating treatment for the prevention of SUDEP highlights the lack of good quality evidence to support interventions with significant risk of bias. There is some evidence to support the protective effect of nocturnal supervision and implementing special precautions such as monitoring systems $[28,52]$. There is limited low level evidence to support the effectiveness of measures such as safety pillows ${ }^{61}$, but there is little evidence to support selective serotonin reuptake inhibitors (SSRIs), or adenosine and opiate substances-despite a theoretical underpinning to their potential benefit in reducing the risk of SUDEP [52]. Systems that identify post-ictal generalized EEG suppression (suggested as a possible biomarker of SUDEP risk) have been developed and trialled, however identification of a consistent EEG pattern is complex and to date no gold standard recording has been agreed [67].

\subsection{Epilepsy surgery}

Those with ongoing focal seizures and an identified surgical epileptogenic target should be referred for assessment with a view to improving seizure control [52]. Seizure freedom following resective epilepsy surgery is associated with an overall reduction in mortality. For individuals who are not seizure free following surgery there is an increased risk of SUDEP [68]. It has also been showed in a large cohort that for those surgically treated the risk is lower when compared to those with medical therapy [69].

\subsubsection{Vagus Nerve Stimulation (VNS)}

In the treatment resistant epilepsy population the use of VNS for at least two years has been shown to reduce the risk [70]. Overall VNS therapy has been shown to be associated with slightly lower rates of SUDEP in the high risk treatment resistant population [71]. Recent developments have shown how VNS can be linked to ECG recording to identify ictal tachycardia, which will then trigger stimulation of the Vagus nerve [72] but it is not known if this could have an impact on the risk.

\subsubsection{Responsive Nerve Stimulation (RNS)}

RNS is a relatively new intervention licenced for use since 2011. It is considered a promising treatment. The positive effects on seizures is expected due to short term and long term effects on 
neurobiological and genetic expression on brain connectivity and its network architecture [73]. Early trends suggest it has an impact of reducing SUDEPs by reducing seizures [74].

\subsection{Seizure monitoring devices}

A systematic review of seizure detection devices highlights that there is only limited low level evidence available to support their use. The development of such devices is currently in its infancy and specificity levels remain low [75]. There are a wide range of technologies available which are designed to monitor seizure activity. These include bed sensors, (see below) and direct auditory or visual surveillance systems. Many of the devices have been shown to be effective in monitoring convulsions. There is limited evidence to support an assumption that they will reduce SUDEP risk.

\subsubsection{Movement sensors}

There are a number of devices available monitoring seizure activities through different modalities with varying success. Pressure mats are placed under the mattress and have built in sensors to detect movement. The sensitivity of the device to abnormal movements can be adjusted to match the need of the individual and their seizures. When assessed there is a wide variation in success detecting nocturnal seizures, ranging from failure to detect activity to $89 \%$ detection [75]. Another potential problem with the pressure sensors currently available is the high number of false positive results recorded [76]. The use of video monitoring for seizure identification is to date unproven with EEG comparison [77]. There is some evidence that movements observed on infrared monitors correlate with carer reports which may be of clinical relevance [58].

\subsubsection{Physiological parameters}

Numerous physiological parameters have also shown success in identifying seizure activity including heart rate, autonomic functioning, and apnoea. Combining the measurement of multiple physiological parameters into everyday devices and applications may be the future [75]. A review of Non-EEG seizure detection systems suggests that there is a wide range of sensitivity of seizure detection depending upon the device, the seizure type considered, and the parameters measured [78]. More evidence is emerging of the specifically in regard to the applicability and importance of measuring electrodermal activity in relation to seizure activity and its role as a SUDEP biomarker or risk factor which can be monitored using various technologies and wearable smart devices [79].

There are devices available that can detect changes in velocity and motion often referred to as Accelerometers. One of the main positives of such technology is the ability to integrate it with current commercially available and widely used products such as smart watches and smart phones. The evidence available at present comes from small study designs and results are varied. There is a consistent finding of good sensitivity, but not $100 \%$. Specificity levels have generally been found to be much lower [75].

More recently a multi-centre assessment of wearable multimodal convulsive seizures detector compared to video EEG demonstrated that newer technology may provide more accurate seizure detection, and the device is tolerable in everyday use. The device which detects electrodermal activity and accelerometer signals yielded a high sensitivity rate (up to $100 \%$ ), with much improved 
specificity levels on previous investigations- missing no nocturnal seizures [80]. Convulsions are associated with autonomic dysfunction; this may be best detected by measuring electrodermal activity [81].

Heart rate, heart rate variability and ECG morphology have all been shown to be effected by seizure activity, particularly convulsions [82]. The development of a system using an ECG to detect seizure activity has been trialled with good sensitivity [83]. ECG monitoring has also been combined with additional monitoring including accelerometers, video imaging and audio surveillance [84]. The seizure detection rate using a seizure detection algorithm with a portable, wearable ECG for long term seizure detection has been shown to be comparable with hospital ECG. The technology is also available to use such devices in combination with a wearable photoplethysmography [85].

Changes in heart rate, oxygen saturation, and then electrodermal activity during seizures have been shown with the use of a smart watch with integrated finger cuff with plethysmograph [86]. The wearable apnoea detection device (WADD) is a small device suitable for home use which detects episodes of spontaneous apnoea. In a small pilot study of individuals referred for sleep apnoea diagnosis and healthy controls, the device demonstrated good sensitivity and high specificity in comparison to clinical scoring, and good tolerability by the user [86].

Electromyography (EMG) has good sensitivity in detecting convulsions and low numbers of false positive results when compared to video EEG [87]. These preliminary results may lead to the implementation of portable devices available for use in the community that are in development [88].

\subsubsection{Smart technology applications (Apps)}

A number of smart phone or smart watch apps have been developed to help in epilepsy management. These apps can be an important platform for communication and risk assessment, however to date there is little evidence base to their use. One of the main advantages of utilizing such technology platforms is their acceptability to younger generations. The incorporation of sensor technology into smartphone and smartwatch devices that are readily accessible using specifically designed seizure algorithms shows high rates of detection success [89]. Embrace has been developed for the smart watch platform to help individuals monitor their epilepsy and claims to detect GTC seizures. This device combines electrodermal monitoring and accelerometer technology, but also measures heart rate and temperature [90]. The EpiWatch is an app designed specifically for use on the Apple iWatch. This more sophisticated app is aimed at detecting seizure related movements along with monitoring physiological parameters such as heart rate variation.

\subsection{Longitudinal risk assessment}

\subsubsection{SUDEP and Seizure Safety Checklist}

The SUDEP and Seizure Safety Checklist [58] is a freely available evidence based risk assessment tool for clinicians. The items included are regularly updated when new evidence emerges and the checklist covers the risk factors we have discussed in a series of simple questions. The SUDEP and seizure safety checklist can be used in clinical practice to help structure discussion around SUDEP risk for individuals, families, and carers. Implementing the checklist does require the individual to attend clinical reviews and there is evidence that a lack of engagement with services and treatment may be a modifiable risk factor for SUDEP [91]. 


\subsubsection{EpSMon}

EpSMon (epilepsy self-monitor) [92] is a freely available mobile phone app that is based on the SUDEP safety checklist. The EpSMon is a self-administered questionnaire that helps people take ownership of their epilepsy monitoring and advises a risk assessment every 3 months. If risks are identified then educational advice is provided and recommendations are made for the user to contact their general practitioner or specialist for review [93].

\subsubsection{Care Flow - Epilepsy Networks}

This project integrates wearable technology securely with individuals and clinician held mobile solutions for people with epilepsy. The aim is to make care more tailored and timely by focusing scarce specialist resources at the point of need, all as close as possible to the individual - in their hand. The project looks to develop co-production of care records and foster 'smart' follow up based on need [94]. This project is currently being tested across 12 UK NHS sites.

\section{Clinical Guidelines}

The National Institute for Health and Care Excellence (NICE) Clinical Guidelines [95] states that SUDEP risk should be discussed with an individual at the point of diagnosis. This is also recommended on an American Academy of Neurology (AAN) guideline (2017) [53]. The AAN guidelines goes further to recommend that those individuals with ongoing convulsions are actively managed to reduce seizure frequency. It is also recommend that those with frequent nocturnal convulsions should be advised on nocturnal supervision or remote nocturnal monitoring devices. In general clinicians should be discussing the benefit of seizure freedom in terms of SUDEP risk and how this may be best achieved through adherence to medication.

\section{Conclusion}

The evidence shows that some SUDEP risk factors may be modifiable. The clinical complexities and multifactorial risk factors that include aspects of the biological, psychological and social aspects of epilepsy means those providing appropriate interventions that are acceptable to individuals can be challenging. The most important factor in managing SUDEP risk is seizure control. To work towards a goal of seizure freedom a collaborative approach is required between the individual and all involved healthcare professionals. Many SUDEP cases had limited contact with primary care and even fewer have undergone a specialist epilepsy review in the year prior to death. There is a clear need to ensure that the delivery of service to this high risk group is appropriate. There should also be clear pathways to link with mental health services to manage any psychiatric co-morbidity associated with epilepsy [91] .

\section{Expert commentary}


Several attempts have been made to rank the most relevant risk factors for SUDEP $[35,36]$. It is likely that SUDEP is the result of an accumulation or cumulative effect of multifactorial risk factors and circumstances, most likely triggered by the peri-ictal concurrence of a number of predisposing and precipitating factors. Further there may be confounding factors, some unmeasured, contributing to the change in risk factor status. Some of the associated risk factors may be modifiable with good seizure management and risk assessment.

\section{Access-}

It is important that people have access to appropriate care as and when required across primary, secondary and tertiary specialist services.

\section{Availability-}

Within services clinicians must have the right skill set and level of expertise to manage complex clinical situations and resources.

\section{Approach-}

Services and clinicians need to offer a consistent approach to care including structured standardised risk assessment procedures. Care should be delivered in a collaborative person centred way to identify and manage any static and modifiable risk factors, considering clinical, social, and psychological parameters putting reasonable adjustments in place to help modify risk.

The course of epilepsy is impacted by a wide range of factors and can vary over time and between individuals significantly. With new research we are gaining greater insight into the mechanisms behind SUDEP and the multifactorial risk factors associated. Therefore, risk assessment and epilepsy management should be person centred, holistic, and consider the most up to date robust evidence available.

\section{Desirable Standard Care [96]}

\section{Seizure Frequency-}

Aim to maximise seizure control particularly in regard to convulsions and nocturnal seizures. This approach should encompass pharmacological and non-pharmacological treatment options, including epilepsy surgery where appropriate. The goal should be to aim to reduce seizures to a frequency of less than 3 per year.

\section{Collateral Risk-}

Clinicians and services should work collaboratively with individuals to deliver person centred information on SUDEP risk. The aim should be to reduce the impact of collateral modifiable risk factors including advocating nocturnal supervision for individuals with nocturnal seizures.

\section{Access to care-}

All should have equitable access to appropriate specialist review and investigations when required. This includes ensuring that reasonable adjustments are made for more vulnerable individuals specifically those with an intellectual disability. 
Individuals should have a thorough assessment of any physical co-morbidities including genetic testing where indicated. There should be liaison with specialist mental health services for any comorbid mental illness.

\section{Minimum Care Standards}

(Practical and acceptable clinical implementation)

- All individuals should be provided with accessible information on SUDEP at the earliest appropriate time.

- Clinicians should work collaboratively with individuals to optimize epilepsy management in the context of treatment resistance considering all aspects of care to reduce seizure frequency.

- Advocate nocturnal vigilance and surveillance where nocturnal seizures are present or when there are transient increases in seizures for whatever reason.

\section{Five-year view}

No future can be envisaged without a reflection on the current challenges. While there has been a major focus on SUDEP as a causation of mortality in recent times more needs to be done. Principle challenges include diagnosis of SUDEP which is predominantly in the legal domain and not primarily in the medical arena. Even though a robust classification for SUDEP exists its use in legal settings is ambivalent and inconsistent. SUDEP remains primarily a first world consideration. There is a lack of awareness of SUDEP in emerging economies where typically epilepsy mortality and morbidity is higher. There is still a debate in many parts of even the developed world on the need to disclose to people with epilepsy the possibility of SUDEP. While in certain countries most significantly the USA and the UK there is an acceptance of the need to discuss SUDEP it is still unclear what information should be given. Further most if not all guidance advice discussion of SUDEP early post diagnosis but fail to take into consideration that epilepsy for a few can be a chronic and lifelong condition. This would then require emphasis on periodic person centred discussion of risk. A challenge is the lack of definitive risk factors to discuss and to tailor them to the strength of evidence. There is no definitive hypothesis of the mechanism of SUDEP to help underpin robust solutions.

Even though challenges exist there has been a drive in recent times to mitigate SUDEP. The next five years would see the use of genetics and brain banks to understand and possibly define certain syndromes hitherto unknown which have vulnerability toward SUDEP. Trials of new antiepileptic drugs and surgery to identify if they can mitigate the risk of SUDEP by better control of Seizures will increasingly occur. There would be development of accessible wearable technology such as watches and apps which would be more sensitive and specific. There would be the development of robust surveillance systems to monitor holistic risk status of individuals over time. More aware populations 
of people with epilepsy and their representatives will be seeking looking to be partners with clinicians to co-produce management and treatment plans. Technology will foster more selfempowerment but could also be a driver for precision response based on change in risk. Health organisations and corporations would look to an integrated care model for delivery of epilepsy care to deliver consistent care and improve outcomes. There needs to be a move to 'educate to protect'. A culture of safety through education and training will spread. Further, education of the legal system and those who influence SUDEP diagnosis is essential. There would be cost effective technological monitoring systems made available to developing countries thus contributing to awareness and reduction of SUDEP.

\section{Key Issues}

- Sudden Unexpected Death in Epilepsy (SUDEP) is regarded as the most common cause of death for people with chronic epilepsy.

- Seizure frequency, in particular convulsions and nocturnal seizures may be the most important modifiable risk factor. Clinicians and epilepsy services should work with individuals in a person centred way to reduce risk by improving seizure control using a holistic approach. This will include addressing all aspects of epilepsy care from a biological, psychological, and sociological view point including assessing co-morbid conditions that may impact upon epilepsy control and overall risk. For people with treatment resistant epilepsy therapeutic intervention should be maximised including the use of anti-epileptic medication, Vagus nerve stimulation (VNS), and epilepsy surgery where indicated.

- There is a role for health professionals involved in the care of people with epilepsy to advocate for the use of nocturnal supervision where individuals are experiencing frequent nocturnal seizures.

- Longitudinal risk may be assessed and then communicated to people through the use of standardised risk assessment tools including the SUDEP and Seizure and Safety Checklist. There is also a mobile phone App EpSMon (epilepsy self-monitor) based upon the SUDEP and seizure Safety check list. EpSMon is a freely available self-administered questionnaire that helps the person to manage their own risk and seek advice where appropriate.

- Technological advancements have allowed for the development of sensitive monitoring devices of various physiological parameters associated with SUDEP risk. This technology can be applied to smart devices used in everyday life. This may be the future of monitoring seizure activity and risk factors. There is still need for more robust evidence on the efficacy and safety of such devices.

- SUDEP is a rare event and current evidence informing our views is limited. There is a need for more robust research into this condition, the pathological mechanisms, associated risk factors, identification of biomarkers, and intervention outcomes. 


\section{References: -}

1. De Boer, H.M., Mula, M. and Sander, J.W., (2008). The global burden and stigma of epilepsy. Epilepsy \& behavior, 12(4),540-546

2. Devinsky, O., Spruill, T., Thurman, D et al (2016) Recognizing and preventing epilepsyrelated mortality A call for action. Neurology, 86(8),779-786.

* this paper is a Call for Action paper summarising the current need to recognise amenable mortality in epilepsy

3. Moran, N.F., Poole, K., Bell, G., et al (2004) Epilepsy in the United Kingdom: seizure frequency and severity, anti-epileptic drug utilization and impact on life in 1652 people with epilepsy. Seizure, 13(6),.425-433.

4. Gaitatzis, A., Johnson, A.L., Chadwick, D.W., et al(2004) Life expectancy in people with newly diagnosed epilepsy. Brain, 127(11), 2427-2432.

5. Annegers, J.F. and Coan, S.P., (1999) SUDEP: overview of definitions and review of incidence data. Seizure, 8(6), 347-352.

6. Sander, J.W. and Bell, G.S., (2004) Reducing mortality: an important aim of epilepsy management. J Neurol Neurosurg Psychiatry;75:349-351.

7. Shorvon, S. and Tomson, T., (2011) Sudden unexpected death in epilepsy. The Lancet, 378(9808),2028-2038.

8. Ficker, D.M., So, E.L., Shen, W.K. et al(1998) Population-based study of the incidence of sudden unexplained death in epilepsy. Neurology, 51(5),1270-1274.

9. Holst, A.G., Winkel, B.G., Risgaard, B. etal (2013) Epilepsy and risk of death and sudden unexpected death in the young: a nationwide study. Epilepsia, 54(9), 1613-1620.

10. Thurman, D.J., Hesdorffer, D.C. and French, J.A., (2014) Sudden unexpected death in epilepsy: assessing the public health burden. Epilepsia, 55(10),.1479-1485.

* This paper is important to illustrate the economic cost of SUDEP

11. Tomson, T., Nashef, L. and Ryvlin, P., (2008) Sudden unexpected death in epilepsy: current knowledge and future directions. The Lancet Neurology, 7(11),1021-1031.

12. Lamberts, R.J., Thijs, R.D., Laffan, A. et al(2012) Sudden unexpected death in epilepsy: people with nocturnal seizures may be at highest risk. Epilepsia, 53(2),253-257.

13. Sveinsson, O., Andersson, T., Carlsson, S. et al (2017) The incidence of SUDEP A nationwide population-based cohort study. Neurology, 10-1212.

14. Donner, E.J., Smith, C.R. and Snead, O.C., (2001) Sudden unexplained death in children with epilepsy. Neurology, 57(3),430-434.

15. Ackers, R., Besag, F.M., Hughes, E., et al (2011) Mortality Rates and Causes of Death in Children with Epilepsy Prescribed Antiepileptic Drugs. Drug safety, 34(5), 403-413.

16. Berg, A.T., Nickels, K., Wirrell, E.C. et al 2013. Mortality risks in new-onset childhood epilepsy. Pediatrics, 132(1), pp.124-131.

17. Nashef, L., (1997) Sudden unexpected death in epilepsy: terminology and definitions. Epilepsia, 38(s11). 
18. Annegers, J.F., 1997. United States perspective on definitions and classifications. Epilepsia, 38(s11).

19. Nashef, L., So, E.L., Ryvlin, P. and Tomson, T., (2012) Unifying the definitions of sudden unexpected death in epilepsy. Epilepsia, 53(2), 227-233.

** Very important paper which provides the current diagnostic framework for SUDEP

20. Massey, C.A., Sowers, L.P., Dlouhy, B.J. (2014) Mechanisms of sudden unexpected death in epilepsy: the pathway to prevention. Nature Reviews Neurology, 10(5),271-282.

21. Shmuely, S., Van der Lende, M., Lamberts, R.J., et al (2017) The heart of epilepsy: current views and future concepts. Seizure, 44, 176-183.

22. Nashef, L., Walker, F., Allen, P.et al(1996) Apnoea and bradycardia during epileptic seizures: relation to sudden death in epilepsy. J Neurol Neurosurg Psychiatry, 60(3), .297300.

23. Bateman, L.M., Li, C.S. and Seyal, M., (2008) Ictal hypoxemia in localization-related epilepsy: analysis of incidence, severity and risk factors. Brain, 131(12), 3239-3245.

24. Devinsky, O., Hesdorffer, D.C., Thurman, D.J. et al(2016) Sudden unexpected death in epilepsy: epidemiology, mechanisms, and prevention. The Lancet Neurology, 15(10), 1075-1088.

25. Surges, R. and Sander, J.W., (2012) Sudden unexpected death in epilepsy: mechanisms, prevalence, and prevention. Current opinion in neurology, 25(2), 201-207.

26. Ryvlin, P., Nashef, L., Lhatoo, S.D. et al(2013) Incidence and mechanisms of cardiorespiratory arrests in epilepsy monitoring units (MORTEMUS): a retrospective study. The Lancet Neurology, 12(10), 966-977.

27. Nashef, L., Fish, D.R., Garner, S. et al(1999) Sudden death in epilepsy: a study of incidence in a young cohort with epilepsy and learning difficulty. Epilepsia, 36(12), 1187-1194.

28. Langan, Y., Nashef, L. and Sander, J.W., (2005) Case-control study of SUDEP. Neurology, 64(7), 1131-1133.

29. Langan, Y., (2000) REVIEW Sudden unexpected death in epilepsy (SUDEP): risk factors and case control studies. Seizure, 9(3),179-183.

30. Bateman LM, Li CS, Seyal M. Ictal hypoxemia in localization-related epilepsy: analysis of incidence, severity and risk factors. Brain. 2008;131:3239-3245

31. Nashef $L$, Walker F, Allen $P$, et al. Apnoea and bradycardia during epileptic seizures: relation to sudden death in epilepsy. J Neurol Neurosurg Psychiatry. 1996;60:297-300

32. Goldman AM. Mechanisms of sudden unexplained death in epilepsy. Curr Opin Neurol 2015;28:166-174.

** An important paper which summarises the current thinking on the mechanisms of SUDEP

33. Lotufo PA, Valiengo L, Bensenor IM et al. A systematic review and meta-analysis of heart rate variability in epilepsy and antiepileptic drugs. Epilepsia. 2012;53:272-282.

34. Hodges MR, Wehner M, Aungst J et al Transgenic mice lacking serotonin neurons have severe apnea and high mortality during development. J Neurosci. 2009 Aug 19;

29(33):10341-9 
35. Hesdorffer, D.C., Tomson, T., Benn, E. et al (2011) Combined analysis of risk factors for SUDEP. Epilepsia, 52(6), 1150-1159.

36. Tomson, T., Surges, R., Delamont, R. et al (2016) Who to target in sudden unexpected death in epilepsy prevention and how? Risk factors, biomarkers, and intervention study designs. Epilepsia, 57(S1), 4-16.

** Important paper which brings to gether information on risk factors from the different studies till date on SUDEP.

37. Ficker, D.M., So, E.L., Shen, W.K. et al (1998) Population-based study of the incidence of sudden unexplained death in epilepsy. Neurology, 51(5), 1270-1274.

38. Walczak, T.S., Leppik, I.E., D'amelio, M. et al (2001) Incidence and risk factors in sudden unexpected death in epilepsy A prospective cohort study. Neurology, 56(4), 519-525.

39. Kiani, R., Tyrer, F., Jesu, A. et al (2014) Mortality from sudden unexpected death in epilepsy (SUDEP) in a cohort of adults with intellectual disability. Journal of Intellectual Disability Research, 58(6),508-520.

40. Shankar, R., Walker, M., McLean, B. et al (2016) Steps to prevent SUDEP: the validity of risk factors in the SUDEP and seizure safety checklist: a case control study. Journal of neurology, 263(9), 1840-1846.

* This paper showcases the possibility of structured risk factor scoring to evidence change in risk

41. Goldman, A.M., Behr, E.R., Semsarian, C. et al (2016) Sudden unexpected death in epilepsy genetics: Molecular diagnostics and prevention. Epilepsia, 57(S1), 17-25.

42. Tu, E., Bagnall, R.D., Duflou, J et al (2011) Post-mortem review and genetic analysis of sudden unexpected death in epilepsy (SUDEP) cases. Brain Pathology, 21(2), 201-208.

43. Bagnall, R.D., Crompton, D.E., Petrovski, S. et al (2016) Exome-based analysis of cardiac arrhythmia, respiratory control, and epilepsy genes in sudden unexpected death in epilepsy. Annals of neurology, 79(4), 522-534.

44. Dravet, C., (2011) The core Dravet syndrome phenotype. Epilepsia, 52(s2),.3-9.

45. Claes, L., Del-Favero, J., Ceulemans, B. et al (2001) De novo mutations in the sodiumchannel gene SCN1A cause severe myoclonic epilepsy of infancy. The American Journal of Human Genetics, 68(6), 1327-1332.

46. Delogu, A.B., Spinelli, A., Battaglia, D. et al (2011) Electrical and autonomic cardiac function in patients with Dravet syndrome. Epilepsia, 52(s2), 55-58.

47. Kalume, F., Westenbroek, R.E., Cheah, C.S. et al (2013) Sudden unexpected death in a mouse model of Dravet syndrome. The Journal of clinical investigation, 123(4), 1798.

48. Sakauchi, M., Oguni, H., Kato, I. et al (2011) Retrospective multiinstitutional study of the prevalence of early death in Dravet syndrome. Epilepsia, 52(6),1144-1149.

49. Shmuely, S., Sisodiya, S.M., Gunning, W.B. et al (2016) Mortality in Dravet syndrome: a review. Epilepsy \& Behavior, 64,69-74.

50. DeGiorgio, C.M., Markovic, D., Mazumder, R. et al(2017) Ranking the Leading Risk Factors for Sudden Unexpected Death in Epilepsy. Frontiers in neurology, 8, .473.

51. Tomson, T., Walczak, T., Sillanpaa, M.et al (2005) Sudden unexpected death in epilepsy: a review of incidence and risk factors. Epilepsia, 46(s11), 54-61. 
52. Maguire, M.J., Jackson, C.F., Marson, A.G. et al (2016) Treatments for the prevention of Sudden Unexpected Death in Epilepsy (SUDEP). Cochrane Database Systematic Review; 7 : CD011792

**A important paper critically analysing current interventions for SUDEP

53. Harden, C., Tomson, T., Gloss, D. et al (2017) Practice guideline summary: Sudden unexpected death in epilepsy incidence rates and risk factors Report of the Guideline Development, Dissemination, and Implementation Subcommittee of the American Academy of Neurology and the American Epilepsy Society. Neurology, 88(17), 1674-1680.

** Latest good practice guidance on SUDEP and its communication for the USA

54. Liebenthal, J.A., Wu, S., Rose, S. et al (2015) Association of prone position with sudden unexpected death in epilepsy. Neurology, 84(7), 703-709.

55. Shmuely, S., Surges, R., Sander, J.W. et al (2016) Prone sleeping and SUDEP risk: The dynamics of body positions in nonfatal convulsive seizures. Epilepsy \& Behavior, 62,176179.

56. Nilsson, L., Farahmand, B.Y., Persson, P.G. et al (1999) Risk factors for sudden unexpected death in epilepsy: a case control study. The Lancet, 353(9156),888-893.

57. Tomson, T. and Kennebäck, G., (1997) Arrhythmia, heart rate variability, and antiepileptic drugs. Epilepsia, 38(s11).

58. Shankar, R., Cox, D., Jalihal, V. et al (2013) Sudden unexpected death in epilepsy (SUDEP): development of a safety checklist. Seizure, 22(10),812-817.

** Highlights the importance of person centred evidence based communication of risk to foster patient empowerment and shared decision making on risk issues

59. Lip, G.Y., and Brodie, M.J (1992), Sudden death in epilepsy: an avoidable outcome? Journal of the Royal Society of Medicine, 85 609-611.

60. Opeskin, K., Burke, M.P., Cordner, S.M. et al (1999) Comparison of antiepileptic drug levels in sudden unexpected deaths in epilepsy with deaths from other causes. Epilepsia, 40(12), 1795-1798.

61. Ryvlin, P., Cucherat, M. and Rheims, S., (2011) Risk of sudden unexpected death in epilepsy in patients given adjunctive antiepileptic treatment for refractory seizures: a meta-analysis of placebo-controlled randomised trials. The Lancet Neurology, 10(11),961968.

62. Ridsdale, L., Charlton, J., Ashworth, M. et al (2011), Epilepsy mortality and risk factors for death in epilepsy: a population-based study, British Journal of General Practice, 61 (586) 271-278.

* Important paper looking at holistic risk factors in the community not just focusing on Seizure related issues

63. Faingold, C.L., Tupal, S. and Randall, M., (2011) Prevention of seizure-induced sudden death in a chronic SUDEP model by semichronic administration of a selective serotonin reuptake inhibitor. Epilepsy \& Behavior, 22(2),186-190. 
64. Monté, C.P.J.A., Arends, J.B.A.M., Tan, I.Y. et al(2007) Sudden unexpected death in epilepsy patients: risk factors: a systematic review. Seizure, 16(1), .1-7.

65. Dupuis, M., Van Rijckevorsel, K., Evrard, F. et al(2012) Takotsubo syndrome (TKS): a possible mechanism of sudden unexplained death in epilepsy (SUDEP). Seizure, 21(1), 5154.

66. Catcheside, P.G., Mohtar, A.A. and Reynolds, K.J., (2014) Airflow resistance and CO 2 rebreathing properties of anti-asphyxia pillows designed for epilepsy. Seizure, 23(6), 462467.

67. Theeranaew, W., McDonald, J., Zonjy, B. et al (2017) Automated Detection of Post-ictal Generalized EEG Suppression. IEEE Transactions on Biomedical Engineering.

68. Sperling, M.R., Feldman, H., Kinman, J. et al (1999) Seizure control and mortality in epilepsy. Annals of neurology, 46(1), .45-50.

69. Sperling, M.R., Barshow, S., Nei, M. et al (2016) A reappraisal of mortality after epilepsy surgery. Neurology, 86(21), 1938-1944.

70. Annegers, J.F., Coan, S.P., Hauser, W.A. et al (2000) Epilepsy, Vagal Nerve Stimulation by the NCP System, All-Cause Mortality, and Sudden, Unexpected, Unexplained Death. Epilepsia, 41(5), 549-553.

71. Granbichler, C.A., Nashef, L., Selway, R. et al (2015) Mortality and SUDEP in epilepsy patients treated with vagus nerve stimulation. Epilepsia, 56(2),291-296.

72. Schneider, U.C., Bohlmann, K., Vajkoczy, P. et al (2015) Implantation of a new Vagus Nerve Stimulation (VNS) Therapy ${ }^{\circledR}$ generator, AspireSR ${ }^{\circledR}$ : considerations and recommendations during implantation and replacement surgery-comparison to a traditional system. Acta neurochirurgica, 157(4),.721-728.

73. Thomas GP, Jobst BC. Critical review of the responsive neurostimulator system for epilepsy (2015). Medical Devices (Auckland, NZ). 2015; 8:405-411.

74. Devinsky O, Friedman D, Duckrow RB, et al. (2018) Sudden unexpected death in epilepsy in patients treated with brain-responsive neurostimulation. Epilepsia.00:1-7

75. Jory, C., Shankar, R., Coker, D. et al(2016) Safe and sound? A systematic literature review of seizure detection methods for personal use. Seizure, 36,4-15.

\section{** A systemic review of current surveillance devices excluding EEG and related systems used by family and carers for people with epilepsy.}

76. Carlson, C., Arnedo, V., Cahill, M. et al (2009) Detecting nocturnal convulsions: efficacy of the MP5 monitor. Seizure, 18(3),225-227.

77. Cuppens, K., Chen, C.W., Wong, K.B.Y. et al(2012). Integrating video and accelerometer signals for nocturnal epileptic seizure detection. In Proceedings of the 14th ACM international conference on Multimodal interaction (161-164). ACM.

78. Van de Vel, A., Cuppens, K., Bonroy, B. et al (2016) Non-EEG seizure detection systems and potential SUDEP prevention: State of the art: Review and update. Seizure, 41,141153.

79. Picard, R.W., Migliorini, M., Caborni, C. et al(2017) Wrist sensor reveals sympathetic 
hyperactivity and hypoventilation before probable SUDEP. Neurology, 89(6), 633-635.

80. Onorati, F., Regalia, G., Caborni, C et al (2017) Multicenter clinical assessment of improved wearable multimodal convulsive seizure detectors. Epilepsia, 58(11),1870-1879.

81. Cogan, D., Nourani, M., Harvey, J. et al (2015) August. Epileptic seizure detection using wristworn biosensors. In Engineering in Medicine and Biology Society (EMBC), 2015 37th Annual International Conference of the IEEE (5086-5089). IEEE.

82. Surges, R., Scott, C.A. and Walker, M.C., (2010) Enhanced QT shortening and persistent tachycardia after generalized seizures. Neurology, 74(5), .421-426.

83. Massé, F., Penders, J., Serteyn, A. et al(2010). Miniaturized wireless ECG-monitor for realtime detection of epileptic seizures. In Wireless Health 2010 (111-117). ACM.

84. Sabesan, S. and Sankar, R., (2015) Improving long-term management of epilepsy using a wearable multimodal seizure detection system. Epilepsy \& Behavior, 46,.56-57.

85. Vandecasteele, K., De Cooman, T., Gu, Y. et al(2017) Automated Epileptic Seizure Detection Based on Wearable ECG and PPG in a Hospital Environment. Sensors, 17(10), 2338.

86. Rodriguez-Villegas, E., Chen, G., Radcliffe, J. et al (2014) A pilot study of a wearable apnoea detection device. BMJ open, 4(10), 005299.

87. Cavazos, J., Girouard, M. and Whitmire, L., (2015) Novel ambulatory EMG-based GTC seizure detection device for home $\&$ hospital use (I6-4B). Neurology, 84(14 Supplement), 16-4B.

88. Graves, J., Arjona, J. and Gourraud, P.A., (2017) Wearable electromyogram device associated with disability outcomes in adults with multiple sclerosis (P1.377) Neurology; 88 (16 Supplement)

89. Bojanovsky, V., Byrne, S., Kirwan, P. et al (2017) Evaluation of Fall and Seizure Detection with Smartphone and Smartwatch Devices. In International Conference on Ubiquitous Computing and Ambient Intelligence ( 275-286). Springer, Cham.

90. Poh, M.Z., Loddenkemper, T., Reinsberger, C. et al 2012. Convulsive seizure detection using a wrist-worn electrodermal activity and accelerometry biosensor. Epilepsia, 53(5).

91. Shankar, R., Jalihal, V., Walker, M. et al(2014) A community study in Cornwall UK of sudden unexpected death in epilepsy (SUDEP) in a 9-year population sample. Seizure, 23(5), 382-385.

92. Newman, C., Shankar, R., Hanna, J. et al (2016) Developing an evidence-based epilepsy risk assessment ehealth solution: from concept to market. JMIR research protocols, 5(2).

93. Shankar, R., Newman, C., McLean, B. et al (2015) Can technology help reduce risk of harm in patients with epilepsy? British Journal of General Practice 2015 65(638): 448-449.

94. https://www.kingsfund.org.uk/sites/default/files/media/Rupert Page T3A.pdf (accessed $16 / 12 / 2017)$

95. The National Institute for Health and Care Excellence (NICE). (2012) The epilepsies: the diagnosis and management of the epilepsies in adults and children in primary and secondary care. NICE clinical guideline 137 guidance.nice.org.uk/cg137

*Best practice guidance for SUDEP in the UK 
96. Shankar R, Donner E, McLean B et al 2017 Sudden Unexpected Death in Epilepsy -What Every Neurologist Should Know Epileptic Disorders 19, 1: 1-9

** Important paper which summarises the key issues which primary care physicians and non-epilepsy specialist neurologists should know about SUDEP and how to assess and communicate its risk to patients.

\section{Appendix 1:}

Unified sudden unexpected death in epilepsy (SUDEP) definition and classification (Nashef et al 2012) [18]

1. Definite SUDEP: Sudden, unexpected, witnessed or unwitnessed, nontraumatic and nondrowning death, occurring in benign circumstances, in an individual with epilepsy, with or without evidence for a seizure and excluding documented status epilepticus (seizure duration $\geq 30$ min or seizures without recovery in between), in which post-mortem examination does not reveal a cause of death

1a. Definite SUDEP Plus: Satisfying the definition of Definite SUDEP, if a concomitant condition other than epilepsy is identified before or after death, if the death may have been due to the combined effect of both conditions, and if autopsy or direct observations/recordings of terminal event did not prove the concomitant condition to be the cause of death

2. Probable SUDEP/Probable SUDEP Plus: Same as Definite SUDEP but without autopsy. The victim should have died unexpectedly while in a reasonable state of health, during normal activities, and in benign circumstances, without a known structural cause of death

3. Possible SUDEP: A competing cause of death is present

4. Near-SUDEP/Near-SUDEP Plus: A patient with epilepsy survives resuscitation for more than 1 hour after a cardiorespiratory arrest that has no structural cause identified after investigation

5. Not SUDEP: A clear cause of death is known

6. Unclassified: Incomplete information available; not possible to classify

If a death is witnessed, an arbitrary cut off of death within $1 \mathrm{~h}$ from acute collapse is suggested

\section{Appendix 2}

Some observed physiological variations at time of a convulsion: 
Arterial hypertension, increased cerebral venous pressure, decreased Arterial PO2, increased Arterial PCo2 and Central venous PCo2, increased cerebral blood flow, Hyperglycaemia, Hyperkalaemia, Haemoconcentration, Lactacidosis, increased Plasma Prolactin, ACTH, GH, TSH and cortisol.

\section{Appendix 3}

Some common characteristics of definite or probable SUDEP and near SUDEP adapted from the MORTEMUS study [26]

- Convulsion preceding event

- Nocturnal events

- Prone position

Table 1: Pooling the data: The positives and negatives when considering results

(adapted from Hesdorffer et al 2011; Tomson et al, 2016 ${ }^{31}$ )

\begin{tabular}{l|l}
\hline \multicolumn{1}{c|}{ Positives } & \multicolumn{1}{c}{ Negatives } \\
\hline Increase sample size and statistical significance & Methodological disparities \\
Increase generalisability & $\begin{array}{l}\text { Heterogeneity of sample population: } \\
\text {-Variation in sample size } \\
\text {-Identification of cases variable } \\
\text {-Restrictive inclusion criteria } \\
\text {-Sample setting variability }\end{array}$ \\
$\begin{array}{l}\text { Case and control definitions consistent between } \\
\text { investigations }\end{array}$ & Matching variables and potential confounders \\
Risk factor ascertainment consistent & $\begin{array}{l}\text { Differing ratio of cases to controls between } \\
\text { studies }\end{array}$ \\
$\begin{array}{l}\text { Definition of definite or probable SUDEP } \\
\text { consistent }\end{array}$ & $\begin{array}{l}\text { Lack of complete information of important } \\
\text { variable including age }\end{array}$ \\
Consistent control group allocation (from same & Lack of consistent risk factor examination across \\
\hline
\end{tabular}


